\title{
OS EFEITOS DO TOQUE TERAPÊUTICO NA SÍNDROME PRÉ-MENSTRUAL
}

\section{THE EFFECTS OF THERAPEUTIC TOUCH ON PREMENSTRUAL SYNDROME}

\section{RESUMO}

O estudo teve por finalidade verificar a eficácia do Toque Terapêutico (TT ou método Krieger-Kunz) sobre os sintomas da Síndrome Pré-Menstrual (SPM) que acometem muitas mulheres. Tratou-se de um estudo piloto e a amostra foi composta por vinte graduandas de Enfermagem da Universidade de São Paulo que atenderam aos critérios de inclusão do projeto e se submeteram a uma sessão semanal de TT por três meses. Foi realizada uma análise das proporções com o teste t-pareado relacionada às variáveis que corresponderam aos sintomas do Índice de Blatt e Kupperman, coletadas antes e depois das intervenções com a terapia complementar. $\mathrm{O}$ resultado encontrado foi $p=0,000$, o que indica uma diferença significativa antes e depois da intervenção, demonstrando a eficácia do TT na diminuição dos sintomas da SPM com mudanças significativas entre o momento zero e o momento final. Neste estudo a intervenção com o Toque Terapêutico trouxe alívio à Síndrome Pré-Menstrual.

\section{PALAVRAS-CHAVE}

\author{
Enfermagem. \\ Terapias Complementares. \\ Toque Terapêutico. \\ Síndrome Pré-Menstrual.
}




\begin{abstract}
The study aimed to verify the effectiveness of Therapeutic Touch (TT or Krieger-Kunz Method) on the symptoms of premenstrual syndrome (PMS) that affects many women. It was a pilot study and the sample was composed of twenty nursing students from USP who met the inclusion criteria of the project and underwent a weekly session of TT for three months. The researchers conducted an analysis with the paired t-test related to the variables that corresponded to the symptoms of the Blatt and Kupperman Index, and they were collected before and after the interventions with the complementary therapy. The result found was $\mathrm{p}=0,000$; this indicates a significant difference before and after the intervention, and it demonstrates the effectiveness of TT in reducing the symptoms of PMS with significant changes between the zero time and the final moment. The intervention with Therapeutic Touch provided relief on premenstrual syndrome in this study.
\end{abstract}

KEYWORDS: Nursing, Complementary Therapies, Therapeutic Touch, Premenstrual Syndrome.

\section{INTRODUÇÃO}

A teoria quântica afirma que a realidade do ser humano consiste em campos de energia, onde o campo energético do homem encontra-se interagindo com o que o rodeia, isto é, com o ambiente e também com outro ser humano ${ }^{(1)}$. Essa constante interação exerce grande influência na vida e no cotidiano das pessoas. "At the split second of critical mass, in a nanosecond, or in a wink, it can change into a thousand-and-one things and then change again and again"(2).

O modo de pensar cartesiano, tradicional, traz a explicação de que tudo é separado de tudo. Cientistas em geral fragmentam sociedades, culturas e até pessoas, com a finalidade de analisar unidades mínimas da natureza, sendo considerado este o único modo para entender o funcionamento das coisas. Porém, com a constatação de que o ser humano é um ser energético, surge uma visão diferente da convencional, ou seja, a explicação dos fenômenos que conhecemos pode ser encontrada se os analisarmos como um todo, onde um aspecto e uma dimensão estão sempre interagindo com outra(s). Trata-se de uma visão holística ${ }^{(3)}$.

A enfermagem holística vem emergindo como uma grande prática profissional. Rogers (1970) desenvolveu a teoria do ser humano unitário ${ }^{(3)}$, propondo que a pessoa não deve ser analisada como uma simples máquina, mas ser avaliada e tratada observando-se todos os componentes (físico, mental e social). Os seres humanos e outros organismos funcionam como unidades completas e integradas e não como um agregado de partes separadas $^{(3)}$.

Baseada na constatação de que o ser humano é um sistema de energia e na visão holística do cuidar, Dolores Krieger (1996) desenvolve uma interpretação moderna da antiga técnica de imposição de mãos $^{(1)}$. Esta doutora da enfermagem, juntamente com Dora Kunz, elaborou uma visão contemporânea desta prática, afirmando que não há ligação com crenças religiosas, pois suas técnicas foram construídas após várias pesquisas e tendo, portanto, embasamento científico ${ }^{(2)}$.

O Toque Terapêutico (TT ou Método Krieger-Kunz) consiste na repadronização do campo energético do paciente, onde quem o aplica tem como objetivo harmonizá-lo e equilibrá-lo. A denominação TT aparenta ser inadequada de início, pois consiste em um "toque sem toque"(4), não havendo necessariamente um toque pele-a-pele. Porém, ao usar conscientemente as mãos a fim de modular o campo energético, seu nome torna-se apropriado.

Essa terapia requer alguns processos na sua aplicação, para que seja eficaz e não prejudique o terapeuta. Suas principais etapas são centralização, diagnóstico, reequilíbrio da energia e avaliação final ${ }^{(1,4,5)}$. 
Atualmente várias pesquisas sobre seus efeitos foram realizadas, destacando-se a de Gomes et al. ${ }^{(6)}$, que analisa seus efeitos sobre a ansiedade em estudantes de graduação; a de Sá ${ }^{(4)}$, que teve por finalidade avaliar a aplicação do Toque Terapêutico em mulheres portadoras de câncer de mama sob tratamento quimioterápico, mostrando resultados favoráveis ao uso desta ferramenta; o ensaio clínico de Marta et al. ${ }^{(7)}$, que aborda a efetividade do TT sobre a dor, depressão e sono em pacientes com dor crônica, apresentando uma mudança estatisticamente significativa após a abordagem com essa terapia.

Estudos revelam alterações fisiológicas quando utilizamos a imposição das mãos, como mudanças em parâmetros hematológicos e imunológicos, por exemplo ${ }^{(8)}$.

Dentre os distúrbios que exercem influência na saúde da população feminina, temos os sintomas pré-menstruais como grandes causadores de incômodos no âmbito emocional e/ou físico. Atualmente denominada Síndrome Pré-menstrual (SPM), corresponde ao conjunto de sinais e sintomas que surgem após o período ovulatório e que desaparecem com o início do fluxo menstrual ${ }^{(10)}$. A SPM é definida como a ocorrência repetida de irritabilidade ou depressão e fadiga durante a fase lútea do ciclo da mulher, acompanhadas de cefaléia e/ou sensação de edema e hipersensibilidade mamária, no abdome e extremidades ${ }^{(9)}$. O termo Síndrome Pré-Menstrual deve ser utilizado em mulheres que relatam pelo menos um desses sintomas comportamentais e um destes sintomas físicos.

Trata-se de um distúrbio comum, que acomete aproximadamente $40 \%$ das mulheres em idade reprodutiva, e engloba uma enorme variedade de sintomas (mais de 150 relacionados a diversos órgãos e sistemas) ${ }^{(11)}$. Geralmente os sintomas aumentam de modo gradual durante a fase lútea, fazem um pico imediatamente antes das menstruações e depois entram em rápido declínio nos primeiros dias após o início da menstruação $0^{(9)}$.

Sua etiologia é formada por inúmeros fatores e teorias, tais como disfunções hormonais, alterações no sistema renina/angiotensina e vasopressina, serotonina, deficiências de vitaminas, entre outros ${ }^{(10)}$.

A respeito do tratamento, não há uma padronização. Muitos profissionais adotam medidas farmacológicas, tais como tratamento hormonal, antidepressivos, ansiolíticos, benzodiazepínicos e diuréticos, com a finalidade de atenuar os sintomas ${ }^{(11)}$. Porém, há alguns problemas relacionados à utilização de fármacos, por causarem efeitos colaterais e muitos não serem de fácil acesso a toda população (alto custo).

Com essa reflexão é que foi elaborado o presente projeto de pesquisa, pois a utilização de uma prática não farmacológica, bastante estudada no exterior, pode ser útil nesses quadros clínicos. $\mathrm{O}$ Toque Terapêutico, ressalta-se, possui várias vantagens, dentre elas é uma terapia não invasiva e de baixo custo $^{(5,6)}$. Essa terapia, dentre outras holísticas, recebe o apoio do Ministério da Saúde, que implantou a Política Nacional da Medicina Natural e Práticas Complementares (PNPIC) no Sistema Único de Saúde ${ }^{(12)}$, e estimula estudos e pesquisas nessa linha integrativa.

Também reforça essa proposta o fato do Conselho Federal de Enfermagem (COFEN), por meio da Resolução 197/97, reconhecer as terapias alternativas/complementares como uma qualificação e/ ou especialidade do profissional de enfermagem ${ }^{(13)}$. Isso denota uma tendência do crescimento das Práticas Alternativas e Complementares para esta profissão em particular, tanto nos setores de educação como no serviço público e privado ${ }^{(14)}$.

Internacionalmente contribui para o reconhecimento e apoio ao uso do TT a North American Nursing Diagnosis Association (NANDA) possuir, em suas classificações, o item Campo de Energia Perturbado ${ }^{(15)}$, em que o terapeuta pode definir características do campo energético e fatores relacionados às mudanças do mesmo.

Krieger (1996) afirma que "embora a Síndrome Pré-Menstrual seja extremamente complicada, não é muito difícil de aliviar com o toque terapêutico"(1:152). Após realizar várias pesquisas em bases de dados reconhecidas, como a Bireme e a EBSCOhost, 
não foram encontrados estudos referentes ao Toque Terapêutico e seus efeitos sobre a SPM.

O objetivo geral do presente estudo é verificar a eficácia do Toque Terapêutico sobre os sintomas da Síndrome Pré-Menstrual. Os objetivos específicos são:

- Caracterizar a amostra investigada em relação às variáveis sócio-demográficas e clínicas.

- Observar, por meio de cada atendimento, a evolução do campo energético das pacientes.

- Verificar, através do Índice de Blatt e Kupperman (adaptado a SPM), se há melhora nos sintomas pré-menstruais.

\section{MATERIAIS E MÉTODO}

Tratou-se de um estudo piloto, realizado na Escola de Enfermagem da Universidade de São Paulo (EEUSP), após aprovação pelo CEP (nº 818/2009) conforme a Resolução do Conselho Nacional de Saúde $n^{\circ}$ 196. As sessões de avaliação/intervenção foram realizadas no Laboratório de Enfermagem da Universidade de São Paulo.

A amostra foi composta por 20 graduandas em Enfermagem da USP. Foram recrutadas as mulheres que atenderam aos seguintes critérios de inclusão: estar regularmente matriculadas na graduação da EEUSP; apresentar ao menos um sintoma comportamental e um físico - irritabilidade, depressão, fadiga, cefaléia, hipersensibilidade mamária, edema - auto-percebidos como relacionados aos sintomas que precedem a menstruação $0^{(9)}$; concordar em participar do estudo, assinando o Termo de Consentimento Livre e Esclarecido.

As estudantes foram recrutadas pela primeira pesquisadora deste estudo, mediante apresentação do objetivo e método da pesquisa.

$\mathrm{Na}$ primeira consulta foram coletados os dados que caracterizam a participante - nome, data de nascimento, idade, peso, altura, estado civil, ano de graduação, antecedentes clínicos e cirúrgicos, e prática de atividade física. Também foram coletados seus dados clínicos - data da última menstruação, regularidade, fluxo, utilização de contraceptivo hormonal, sinais e sintomas referidos no período pré-menstrual, tratamento anterior para o controle dos sintomas.
Cada paciente recebeu uma sessão semanal, durante três meses, realizada pela pesquisadora que possui o curso de Toque Terapêutico - nível 1 (método Krieger-Kunz). Em cada sessão houve uma ficha de atendimento da terapia, onde foram detalhadas as características do campo energético da paciente.

Todas as mulheres deste estudo foram submetidas ao índice de Blatt e Kupperman (adaptado a SPM), ao início da primeira consulta e ao término da última. $\mathrm{O}$ índice original tem como propósito a soma ponderal dos sintomas menopausais ${ }^{(16)}$. Nesta pesquisa ele foi adaptado aos sintomas pré-menstruais mais citados por especialistas ${ }^{(9-11)}$, onde foi utilizada uma pontuação para expressar a intensidade dos mesmos $(0,1,2$ e 3 , que correspondem a ausente, leve, moderado e intenso, respectivamente).

Os dados foram descritos por meio de tabela e gráficos, e foi realizada uma análise das proporções relacionadas às variáveis, calculadas antes (momento zero) e depois (momento final) das intervenções com o TT. As variáveis corresponderam aos sintomas do Índice de Blatt e Kupperman (adaptado a SPM), e aos aspectos clínicos referidos pela paciente no questionário.

A partir dos resultados alguns testes foram utilizados, dentre eles o t-pareado, cuja função é analisar a diferença entre as médias antes e depois da intervenção com o TT; o teste Alpha de Cronbach, com a finalidade de verificar a confiabilidade do instrumento; e o teste de Levene em que foram analisadas as variâncias, que consistem na medida que avalia o quanto as variáveis do instrumento estão dispersas em relação à uma média aritmética, sendo dada pela média dos quadrados dos desvios.

\section{RESULTADOS}

Não houve nenhuma desistência. Como características sócio-demográficas encontramos que a média das idades foi de 23,6 anos. Somente uma voluntária era casada (5\%). Dentre as colaboradoras, nove (45\%) referiram praticar exercícios físicos, sendo as outras onze (55\%) sedentárias. A média dos índices de massa corpórea (IMC) calculados foi de aproximadamente 22, considerado um parâmetro saudável. Somente uma paciente (5\%) encontrava-se no sobrepeso, com IMC de 29,05. 
Quanto às características clínicas relacionadas ao ciclo menstrual das participantes, de acordo com a data da última menstruação (DUM), nove (45\%) mulheres estavam na fase folicular de seus ciclos menstruais no início da coleta de dados, duas (10\%) estavam na fase ovulatória e nove (45\%) na fase lútea. Treze (65\%) afirmaram ter um intervalo regular entre as menstruações tendo, portanto, as outras sete (35\%) um ciclo irregular. Sobre o fluxo menstrual, dezesseis (80\%) referiram ter um fluxo normal, três (15\%) diminuído, e uma (5\%) aumentado. Quanto ao uso de contraceptivo oral, quatorze (70\%) referiram não fazer uso e as outras seis (30\%) fazem uso regularmente. Dezesseis mulheres (80\%) nunca procuraram tratamento para os sintomas da SPM, e as outras quatro (20\%) referiram tratamentos prévios como fármaco manipulado, gamaline, fitoterápico, e até $\mathrm{o}$ anticoncepcional oral, não obtendo resultados satisfatórios.

As variáveis com maior redução foram: Nervosismo (80\%), Cólicas Uterinas (75\%), Crises de Choro (75\%), Edema (70\%), Depressão ou Tristeza (65\%) e Ansiedade (60\%). As outras variáveis correspondem à Cefaléia (50\%), Fadiga (50\%), Hipersensibilidade Mamária (40\%), Alteração do Apetite (onde $30 \%$ correspondem à melhora ou ausência e outros $30 \%$ referem seu aparecimento no momento final da pesquisa).

Seguem os gráficos relacionados aos principais sintomas da SPM que foram comparados no momento zero ou inicial (sinalizado na cor escura) e final (na cor clara), bem como as respectivas médias e os desvios padrão.

Gráfico 1: Comparação do sintoma Nervosismo no momento zero e no final da abordagem com o TT.

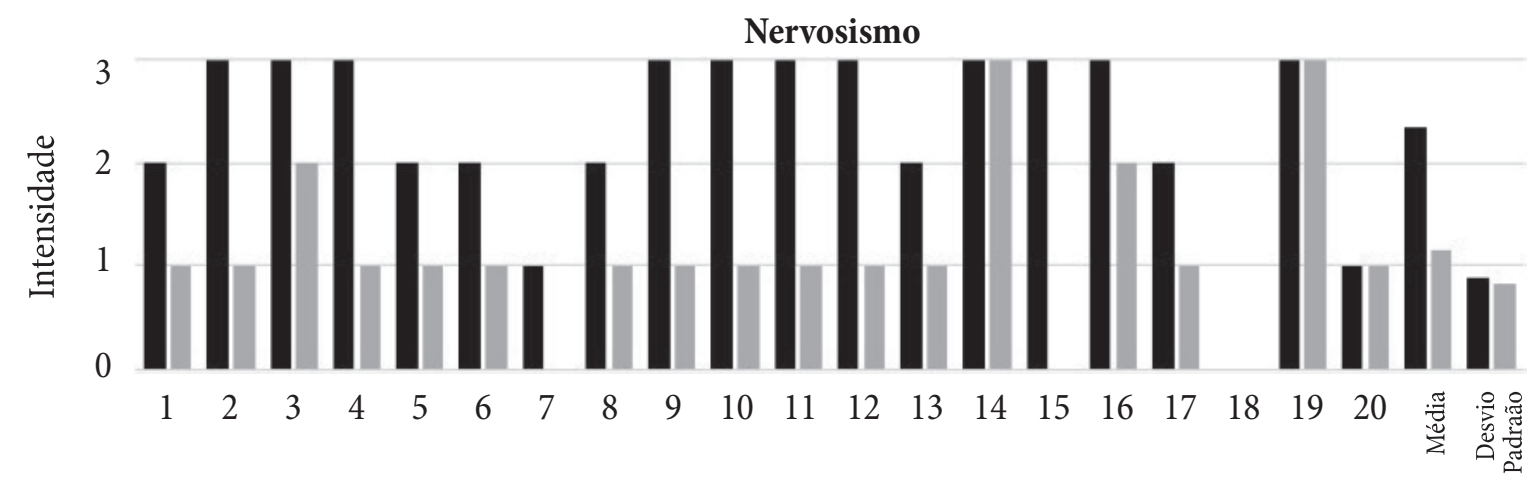

Colaboradoras

Momento zero Momento final

Observa-se que nas pacientes 14, 19 e 20 o nervosismo manteve-se estável, representando $15 \%$ do total das colaboradoras. Já as que referiram melhora ou até ausência do sintoma representam $80 \%$, os 5\% restantes correspondem à paciente 18 , que não sofria desse sintoma.

Gráfico 2: Comparação do sintoma Crises de Choro no momento zero e no final da abordagem com o TT.

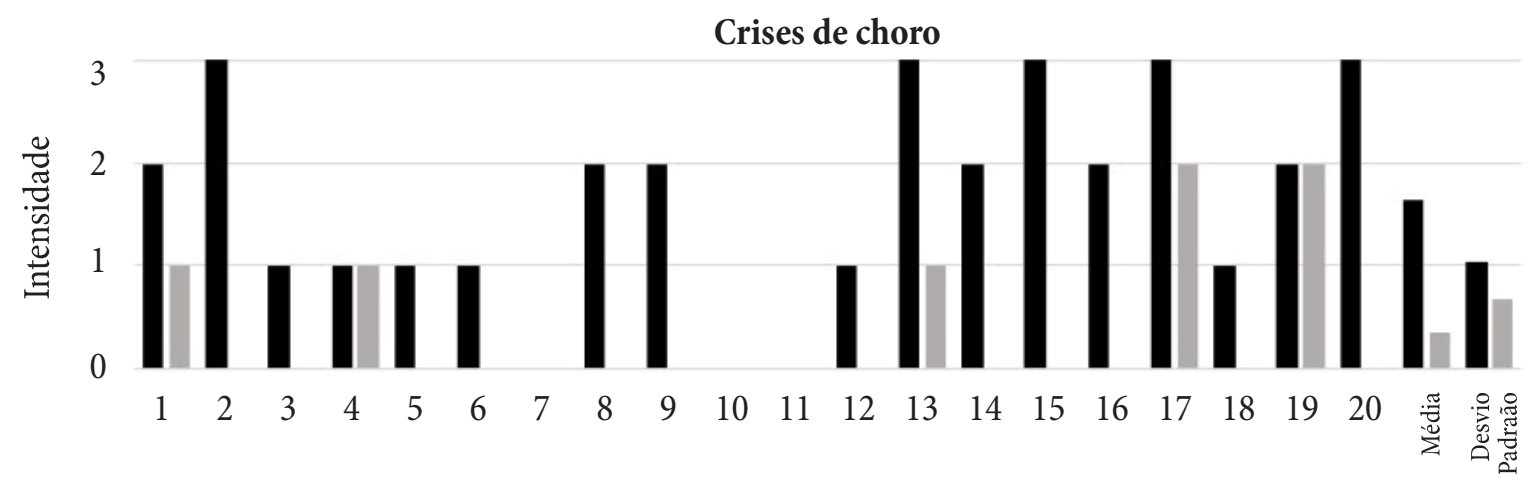

Colaboradoras

Momento zero Momento final 
Nas pacientes 4 e 19 as crises de choro mantiveram-se estáveis, representando $10 \%$ do total das colaboradoras. Já as que referiram melhora ou até ausência do sintoma representam 75\%, sendo os $15 \%$ restantes correspondentes às pacientes que não sofriam desse sintoma.

Gráfico 3: Comparação do sintoma Edema no momento zero e no final da abordagem com o TT.

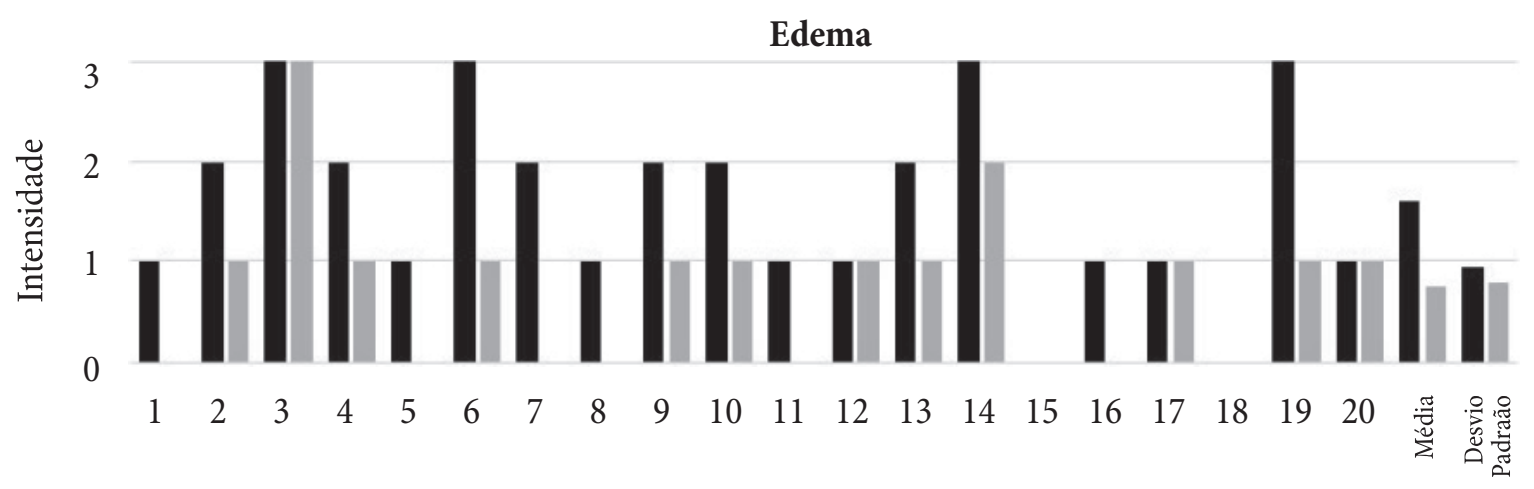

Colaboradoras

Momento zero $\quad$ Momento final

Nas pacientes 3, 12, 17 e 20 o edema manteve-se sintoma representam $70 \%$, sendo os $10 \%$ restantes estável, representando $20 \%$ do total as colaboradocorrespondentes às pacientes que não sofriam desras. Já as que referiram melhora ou até ausência do se sintoma.

Gráfico 4: Comparação do sintoma Cólicas Uterinas no momento zero e no final da abordagem com o TT.

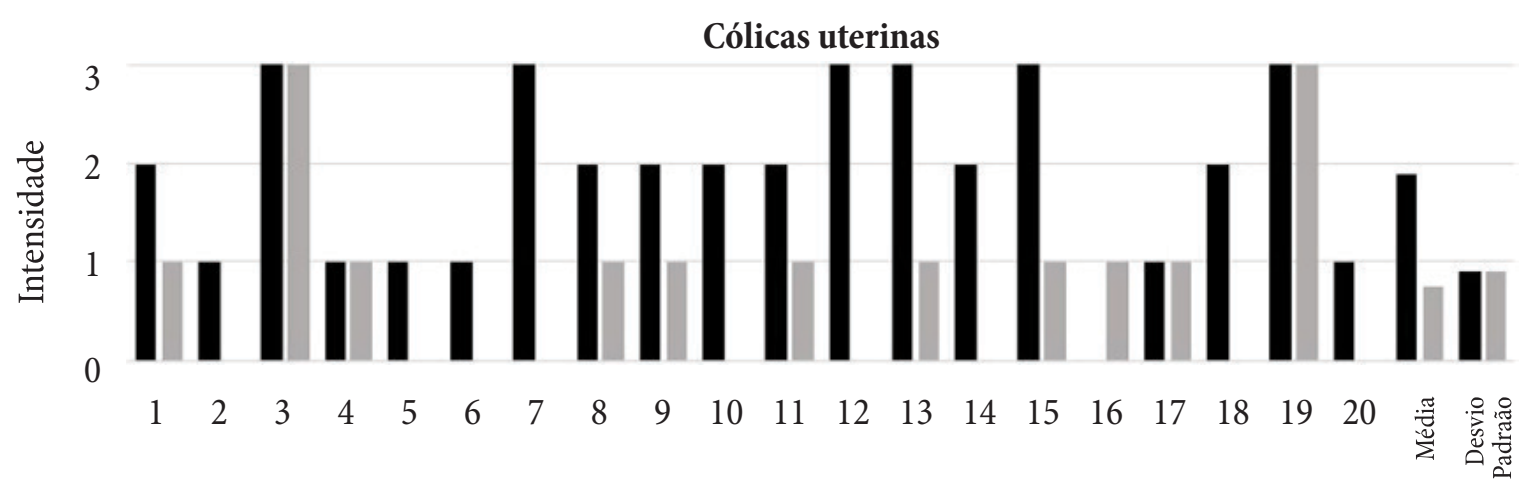

Colaboradoras

Momento zero

Nas pacientes 3, 4, 17 e 19 as cólicas uterinas mantiveram-se estáveis, representando $20 \%$ do total das colaboradoras, as que referiram melhora ou até ausência do sintoma representam $75 \%$, sendo os $5 \%$ restantes correspondentes à mulher 16, que referiu ter notado o aparecimento desse sintoma no momento final. 
Gráfico 5: Comparação do Total dos sintomas no momento zero e no final da abordagem com o TT.

Total

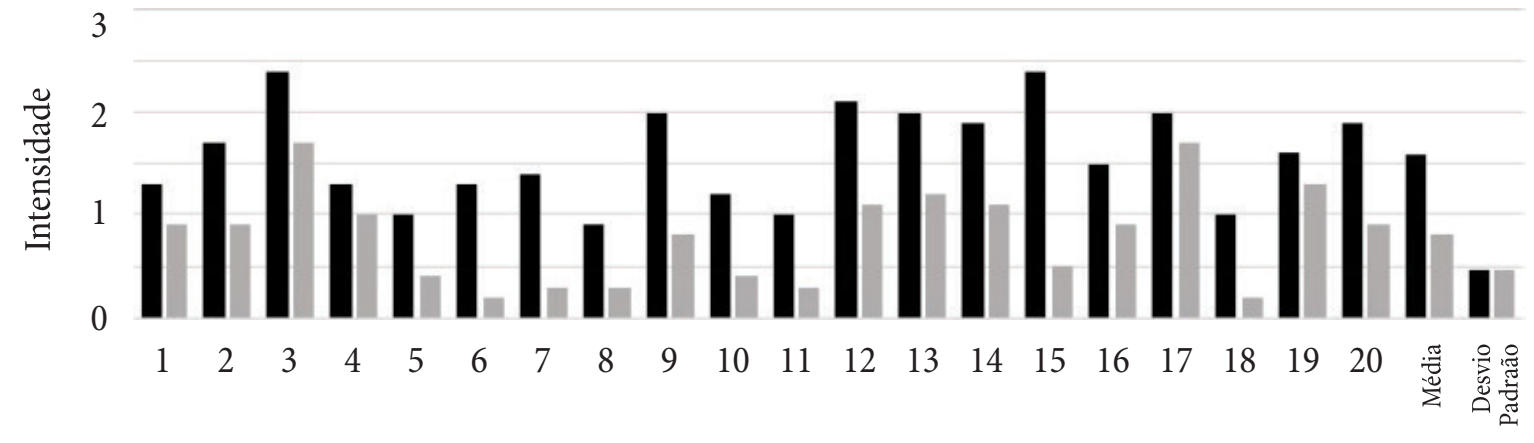

Colaboradoras

Momento zero Momento final

Este gráfico 5 representa o total da intensidade de todos os sintomas das pacientes, e em todas nota-se melhora quando comparados os momentos inicial e final, desde pequenas melhoras como na paciente 17, até grandes melhoras como na paciente 15.

$\mathrm{O}$ valor encontrado no teste t-pareado foi $\mathrm{p}=0,000$, o que indica uma diferença significativa antes e depois da intervenção com a terapia, já que $\mathrm{p}<0,05$.

$\mathrm{O}$ teste de confiabilidade do instrumento (Alpha de Cronbach) após a intervenção com o TT foi de 0,780. Lembramos que para ser considerada uma boa confiabilidade o resultado deve ser no mínimo 0,700 aproximadamente.

Também foram aplicados testes correlacionando a idade das pacientes, seus IMCs e os totais antes e depois dos sintomas expressos no Índice de Blatt e Kupperman (adaptado à SPM). Não foi encontrada correlação significativa, o que indica que o Índice de Massa Corpórea de uma paciente não é influenciado por sua idade e não exerce influência nos sintomas pré-menstruais.

Foi cogitada a hipótese da prática de atividade física influenciar nos resultados. Para isso foram analisadas as variâncias, lembrando-se que quanto maior for uma variância, maior será a dispersão dos dados. $O$ teste de Levene resultou em $p=0,893$, o que indica que as variâncias são iguais. Partindo desta informação pôde-se utilizar o teste-t assumindo variâncias iguais, e p=0,241, indicando que não há diferença na intensidade dos sintomas pré-menstruais entre os grupos com e sem atividade física antes e depois da intervenção com o TT.

Com relação ao uso do contraceptivo oral, utilizou-se a mesma estratégia do item citado anteriormente, e o valor de p para a diferença entre o uso ou não do contraceptivo é $\mathrm{p}=0,525$, ou seja, não existe diferença entre antes e depois da intervenção com a terapia complementar na intensidade dos sintomas entre o grupo que faz uso do contraceptivo e o que não faz.

\section{DISCUSSÃO}

Em todas as sessões de cada uma das pacientes tivemos a oportunidade de aplicar todas as etapas necessárias para aplicações eficazes de TT, desde a centralização da terapeuta, chegando a um diagnóstico, reequilibrando a energia ao aplicar o tratamento, $\mathrm{e}$ fazendo uma avaliação final ${ }^{(1,5,6)}$. Observou-se a evolução do campo energético de cada paciente, e com o decorrer das sessões os campos apresentavam cada vez menos alterações sentidas pela terapeuta e uma melhora gradativa referida pelas colaboradoras. Vale ressaltar que o diagnóstico Campo de Energia Perturbado contido na NANDA pode ser utilizado, em que as características definidoras são percepções de mudanças nos padrões do fluxo de energia, tais como: movimento, mudança de temperatura, mudanças visuais, ruptura do campo e sons ${ }^{(15)}$.

Um fato interessante encontrado após a análise dos dados é que não há diferença na intensidade dos sintomas pré-menstruais entre os grupos com 
e sem atividade física antes e depois da intervenção com o TT, ao contrário do que tem sido dito. Também não foi encontrada uma diferença entre antes e depois da intervenção com a terapia na intensidade dos sintomas entre o grupo que faz uso do contraceptivo oral e o que não faz. Estas informações mostram a necessidade de novos estudos para detalhar o assunto.

Com relação aos sintomas referidos no Índice de Blatt e Kupperman (adaptado a SPM), cabe ressaltar que ele foi adaptado aos sintomas pré-menstruais mais citados por especialistas ${ }^{(9-11)}$, porém 0 que pôde ser observado na coleta de dados é que $90 \%$ das colaboradoras não referiram sofrer do sintoma Insônia, sendo portanto um possível objeto para estudos futuros.

Outro fato intrigante é relacionado à intensidade do sintoma Alteração do Apetite relatada pelas pacientes, onde $30 \%$ do total das mulheres referiram ter notado o aparecimento ou aumento desse sintoma no momento final da intervenção com o TT. A partir daí é levantado um ponto de vista científico para explicar este fato: já que o Toque Terapêutico tem como objetivo reequilibrar o campo energético humano e como um princípio a visão holística no tratamento, uma hipótese para o aparecimento/ aumento desse sintoma é uma busca do organismo dessas pacientes de manter o equilibrio de suas funções na época que antecede a menstruação, e portanto elas podem precisar de alguns nutrientes contidos nos alimentos, o que resulta em uma alteração no apetite. Podemos confirmar este raciocínio com a informação que uma das pacientes relatou informalmente, no momento final da coleta, que durante a época pré-menstrual sua vontade de ingerir carne vermelha aumentava consideravelmente. Isto pode ser associado à perda do componente Ferro na menstruação, e encontrado em grande quantidade no alimento citado pela colaboradora. Todas estas informações podem conduzir a importantes estudos futuros, a fim de coletar e verificar maiores detalhes sobre o assunto.

Embora ainda não existam outros trabalhos específicos sobre o TT e seus efeitos na SPM nas bases de dados, há estudos que abrangem alguns dos distúrbios encontrados também no período pré-menstrual, sendo feita uma associação entre estes sinais e sintomas.

O ensaio clínico de Marta et al. ${ }^{(7)}$ aborda a efetividade do TT sobre a depressão em idosos, em que também observou-se uma mudança significativa entre o momento zero e o momento final, podendo ser feita uma correlação entre a depressão num contexto amplo e na SPM. Neste mesmo estudo constatou-se uma diminuição na intensidade da dor geral de $83,61 \%$, que pode ser comparada aos sintomas de cólicas uterinas e cefaleia, em que a melhora foi de respectivamente $75 \%$ e $50 \%$ das participantes.

O estudo de Santos et al. ${ }^{(17)}$ aborda sobre a dor aguda e sua melhora com o TT, em que os resultados indicam uma alteração benéfica do corpo humano rumo à homeostase, tanto física quanto emocional, sendo associado à alterações que acometem as mulheres na SPM.

O trabalho de Gomes et al.() que analisa os efeitos do TT sobre a ansiedade também pode ser discutido com o presente estudo, visto que este sintoma, além de observado no período pré-menstrual, é encontrado com frequência nos dias atuais. Ambos estudos constataram uma mudança significativa após a aplicação da terapia, sendo relevante desenvolver mais pesquisas na temática.

\section{CONCLUSÃO}

Após a análise dos resultados observa-se melhora como um todo nos sintomas das pacientes, desde pequenas mudanças até grandes melhoras no cotidiano da SPM das pacientes. As variáveis com maior redução foram: Nervosismo (80\%), Cólicas Uterinas (75\%), Crises de Choro (75\%), Edema (70\%), Depressão ou Tristeza (65\%) e Ansiedade (60\%) As outras variáveis correspondem à Cefaléia (50\%), Fadiga (50\%), Hipersensibilidade Mamária (40\%), Alteração do Apetite (30\%).

Por meio desta pesquisa pôde-se aplicar o principio desenvolvido e estudado por Rogers da teoria do ser humano unitário ${ }^{(3)}$, em que o indivíduo foi tratado de uma maneira holística. 
Também observou-se a evolução do campo energético de cada paciente após as aplicações do TT, em que foram constatadas cada vez menos alterações pela terapeuta no decorrer das sessões.

Os dados colhidos no momento zero e no momento final dos encontros com cada paciente foram muito importantes para caracterizar a amostra com relação às variáveis sócio-demográficas e clínicas, assim como para constatar a influência da SPM na vida e no cotidiano dessas mulheres, sendo um grande empecilho no dia-a-dia das mesmas.
O Índice de Blatt e Kupperman (adaptado à SPM) mostrou uma melhora significativa com a diminuição dos sintomas, em geral, quando comparados os momentos inicial e final das doze intervenções com o TT.

Como resultado deste estudo pôde ser constatada a eficácia do Toque Terapêutico, reafirmando a informação de Krieger de que é possível aliviar a SPM com esta prática ${ }^{(1)}$,ressaltando que não é invasiva, de baixo custo e sem os efeitos colaterais das medicações normalmente utilizadas para este problema.

\section{FONTE DE FINANCIAMENTO}

Conselho Nacional de Desenvolvimento Científico e Tecnológico (CNPq).

\section{CONFLITO DE INTERESSES}

Declara não haver

\section{REFERÊNCIAS}

1. Krieger D. O toque terapêutico. Tradutora Lea Passalacqua. São Paulo: Cultrix; 1996.

2. Krieger D. Living the therapeutic touch: healing as a lifestyle. New York (NY): Dodd Mead; 1987.

3. Rogers ME. An introduction to the theoretical basis of nursing. Philadelphia (Ca): Davis; 1970.

4. Sá AC. Aplicação do toque terapêutico em mulheres portadoras de câncer de mama sob tratamento quimioterápico [tese]. São Paulo (SP): Universidade de São Paulo; 2001

5. Savieto RM, Silva MJP, Pozzi DHB, Neto PAF. Ação da água energizada com o toque terapêutico na cicatrização de lesões na pele de camundongos. R Enferm UERJ. 2007; 15(3):423-9.

6. Gomes VM, Silva MJP, Araújo EAC. Efeitos gradativos do Toque Terapêutico na redução da ansiedade de estudantes universitários. Rev Bras Enferm. 2008; 61(6):841-846.

7. Marta IER, Baldan SS, Berton AF, Pavam M, Silva MJP. Efetividade do Toque Terapêutico sobre a dor, depressão e sono em pacientes com dor crônica: ensaio clínico. Rev Esc Enferm USP. 2010; 44(4):1100-1106

8. Oliveira RMJ. Avaliação de efeitos da prática de imposição de mãos sobre os sistemas hematológico e imunológico de camundongos machos. São Paulo, 2003; 75p. Dissertação (Mestrado), FMUSP.

9. Miranda GCV, Miranda S. Alterações do humor relacionadas às variações hormonais: II - síndrome pré-menstrual. Psiquiatria Biológica. 1994; 2(3):57-66

10. Melo NR, Giribela AHG, Giribela CRG, Ricci MD. Síndrome prémenstrual. Rev Bras Med. 2001; 58(esp).
11. López JR, Silva J, Leite E, Abreu AP, Giordano LA. Tratamento psicofarmacológico da síndrome da tensão pré-menstrual: estado atual. Psiquiatria Biológica. 2002; 10(1):16-22.

12. Ministério da Saúde [página na Internet]. Política Nacional de Medicina Natural e Práticas Complementares: um exercício de cidadania. Brasília (DF): Secretaria de Atenção à Saúde; 2004. [acesso em 2009 Jan 28]. Disponível em: http://portal.saude.gov. br/saude/

13. Conselho Federal de Enfermagem [página na Internet]. Legislação: Resolução no 197/97. [citado em 14 de jan 2005; acesso em 2009 Jan 30]. Disponível em: http://www.portalcofen.gov. br/\%5Fnovoportal

14. Barros NF, Tovey P. O ensino das terapias alternativas e complementares em escolas de enfermagem. Rev Gaúcha Enferm. 2007; 28(2):207-14.

15. NANDA. Diagnósticos de Enfermagem da NANDA: definições e classificação. (2012-2014). Porto Alegre (RS): Artmed; 2012

16. Sousa RL, Sousa ESS, Silva JCB, Filizola RG. Fidedignidade do testereteste na aplicação do índice menopausal de Blatt e Kupperman. Rev Bras Ginecol Obstet [online]. 2000 [acesso em 2009 Fev 02]; 22(8):481-7. Disponível em: http://www.scielo.br/scielo. php?script=sci_arttext\&pid=S0100-72032000000800003\&lang=pt

17. Santos AO, Geraldo BLSS, Zani RC, Sá AC. Aplicação do Toque Terapêutico (método Krieger-Kunz) no alívio da dor em uma vítima de politrauma - dignidade humana na assistência de enfermagem. Mundo Saúde. 2004; 28(3):325-332. 